\title{
Detection of Cardiovascular CRP Protein Biomarker Using a Novel Nanofibrous Substrate
}

\author{
Isaac Macwan $1, * \mathbb{(})$ Ashish Aphale ${ }^{2}\left(\mathbb{0}\right.$, Prathamesh Bhagvath ${ }^{3}$, Shalini Prasad ${ }^{4}(\mathbb{C}$ \\ and Prabir Patra 2,5 \\ 1 Department of Electrical and Bioengineering, Fairfield University, Fairfield, CT 06824, USA \\ 2 Department of Biomedical Engineering, University of Bridgeport, Bridgeport, CT 06604, USA; \\ aaphale@my.bridgeport.edu (A.A.); ppatra@bridgeport.edu (P.P.) \\ 3 Department of Biomedical Engineering, Saint Louis University, St. Louis, MO 63103, USA; \\ pbhagvath@slu.edu \\ 4 Department of Bioengineering, University of Texas at Dallas, Richardson, TX 75080, USA; \\ shalini.prasad@utdallas.edu \\ 5 Department of Mechanical Engineering, University of Bridgeport, Bridgeport, CT 06604, USA \\ * Correspondence: imacwan@fairfield.edu; Tel.: +1-203-254-4000
}

Received: 1 June 2020; Accepted: 23 June 2020; Published: 24 June 2020

\begin{abstract}
It is known that different diseases have characteristic biomarkers that are secreted very early on, even before the symptoms have developed. Before any kind of therapeutic approach can be used, it is necessary that such biomarkers be detected at a minimum concentration in the bodily fluids. Here, we report the fabrication of an interdigitated sensing device integrated with polyvinyl alcohol (PVA) nanofibers and carbon nanotubes (CNT) for the detection of an inflammatory biomarker, C-reactive protein (CRP). The limit of detection (LOD) was achieved in a range of $100 \mathrm{ng} \mathrm{mL}^{-1}$ and $1 \mathrm{fg} \mathrm{mL}^{-1}$ in both phosphate buffered saline (PBS) and human serum (hs). Furthermore, a significant change in the electrochemical impedance from $45 \%$ to $70 \%$ (hs) and $38 \%$ to $60 \%$ (PBS) over the loading range of CRP was achieved. The finite element analysis indicates that a non-redox charge transduction at the solid/liquid interface on the electrode surface is responsible for the enhanced sensitivity. Furthermore, the fabricated biosensor consists of a large electro-active surface area, along with better charge transfer characteristics that enabled improved specific binding with CRP. This was determined both experimentally and from the simulated electrochemical impedance of the PVA nanofiber patterned gold electrode.
\end{abstract}

Keywords: biosensors; C-reactive protein; carbon nanotubes; electrospinning; electrochemical impedance

\section{Introduction}

Detection of biomarkers specific to a particular disease is a standard method for diagnosis and can be used for successful detection of various diseases [1]. A number of proteins considered as biomarkers are secreted at an early onset of a disease when the symptoms are yet to develop. A typical source to detect such biomarkers can be a patient's blood sample. C-reactive protein (CRP) has long been used as a marker of systemic inflammation, where the level of CRP increases several hundred fold within hours of an inflammatory occurrence [2] and it is found to be over-expressed in heart disease [3]. Previous research has shown that CRP is dominant in the instigation of several pathogenic pathways that may cause atherosclerosis, a precursor to cardiovascular disease [4]. According to the Center for Disease Control and Prevention, heart attack is the leading cause of death in the US with an estimated 785,000 Americans having a new coronary attack and approximately 470,000 with a recurrent attack [5]. Thus a rapid and ultra-sensitive detection of CRP from a patient's serum sample may be clinically 
significant in diagnosing cardiac events such as acute myocardial infarction. In recent years, the trend in healthcare has been towards developing devices that support personalized medicine with benefits such as faster diagnosis and therapeutic turnaround time.

Nanotechnology offers a wide range of materials that can be used both as biological recognition elements and transducers for a typical biosensor with exceptional levels of sensitivity that can be used to monitor biomarkers from different diseases. Novel properties of nanoscale materials, especially in the form of nanofibers, such as larger surface to volume ratios and size-based confinement of biomolecules, give them the potential to increase the sensitivity of the diagnostic devices by several orders of magnitude [6-11]. This property can be used to detect protein biomarkers such as CRP at very low concentrations and also give an early indication of a heart condition [12,13].

Some of the previous efforts in developing biosensors based on nanofibers involved citric acid-decorated nylon nanofibers for the detection of 3-phenoxybenzoic acid (3-PBA), a common human urinary metabolite [14], and polyvinyl alcohol-co-ethylene (PVA-co-PE)-based nanofibrous membranes for the detection of chloramphenicol (CAP) residues in milk [15]. One of the aspects of polyvinyl alcohol (PVA) and carbon nanotube (CNT)-based nanofibrous biological recognition elements is that they offer spatial confinement similar to the intracellular environment through porosity. The binding affinities and the rate at which a typical self-assembly process takes place can benefit from several physical constraints during the biosensing of macromolecules in vitro [16-18]. The nanotexturing of the sensor platform enables minimization of excluded volume of the biomolecules not participating in the interaction with the biomarker, which is also evident from the increase in the concentration that is analyzed through finite element analysis of diluted species [19]. Furthermore, even though individual fibers may have been laid on the electrode, there are still multiple fibers exhibiting a certain thickness similar to a membrane-like matrix but taking advantage of a higher specific surface area.

One of the major challenges faced by nanomaterial-based biosensors is the sensitivity and reproducibility of the results, which arises mainly because of the extreme difficulty in detecting a small quantity of protein biomarkers in serum samples [20-22]. Hence, determining the concentration of trace biomarkers in a complex mixture is a challenge in patient diagnosis.

Amongst several diagnostic biosensors, most of the conventional ones rely on fluorescent labeling or dyes for gathering the data [23]. Various sensing techniques that are applied to detect biomolecules at low concentrations are electrochemical analysis, surface plasmon resonance, electromagnetic measurements or mechanical actuation. Electrochemical sensors allow a label free detection of biomolecules by detecting and measuring the electrical signal [24-26]. A variety of electrochemical techniques based on integrated devices have been employed for label free and ultra-sensitive detection of different biomarkers. These methods are based on the principles of charge transfer [27-29], radiofrequency [30], complementary metal oxide semiconductors (CMOS) [31], capacitance [32], or impedance [33]. CMOS-based sensors are limited by the complexity of the fabrication technique, which leads to an extremely high cost of the overall biosensor device. In contrast, impedance and capacitance-based measurements are potential techniques for sensing a variety of biomolecules, mainly due to the low power consumption, ease of miniaturization and relatively low cost [34]. It is known that the performance of any biosensor depends on the immobilization of biomolecules on a biocompatible electrode surface. Therefore, the specificity of a biosensor can be increased by increasing the overall specific binding sites on the surface of the electrode. Hence, a biocompatible electrode, with an extremely high surface area that can engender spatial confinement for favorable binding events, would be a preferred platform for antigen-antibody interactions to achieve high sensitivity and specificity.

Here, we report, an extremely sensitive diagnosing platform with an ability to detect CRP concentrations up to $\mathrm{fg} \mathrm{mL}^{-1}$ in both phosphate buffer saline (PBS) and human serum (hs). A microchip with seven interdigitated micro-comb capacitors is integrated with a randomly aligned electrospun PVA nanofibrous mesh. Owing to the random alignment of the nanofibers, the mesh showed high porosity for macromolecule confinement and an extremely small individual fiber diameter $(\sim 250 \mathrm{~nm})$, along with a larger surface area to volume ratio, providing efficient antigen-antibody interactions. 
Furthermore, the signal transduction is found to be chemo-electro ionic, with the protein binding resulting in a modulation of the electrical double layer at the nanofibrous surface. A $45 \%$ change in the measured impedance from the antibody saturation baseline was observed while detecting the lowest detectable dose of $1 \mathrm{fg} \mathrm{mL}^{-1}$. Finally, a change in the oxidation current for a gold substrate with and without PVA/CNT/anti-CRP functionalization provides crucial insights on the charge transfer resistance at the double layer capacitance interface between the metal electrode and the bulk electrolyte. The results obtained through a finite element model are in close agreement with the experimental data.

\section{Materials and Methods}

The nanoweb was fabricated using an electrospinning process (Figure 1a). The electrospinning set up comprises a high voltage power supply and a dual syringe pump unit. A positive electrode from the high voltage power supply is connected to the needle. The nanoweb is collected on a grounded metal collector covered with insulating polyethylene. To produce the PVA-CNT nanocomposite fibrous nanoweb, we applied $15 \mathrm{kV}$ of voltage across $10 \mathrm{~cm}$ distance between the collector and the needle tip with a flow rate of the CNT dispersed polymer solution of $1.5 \mathrm{~mL} / \mathrm{h}$.

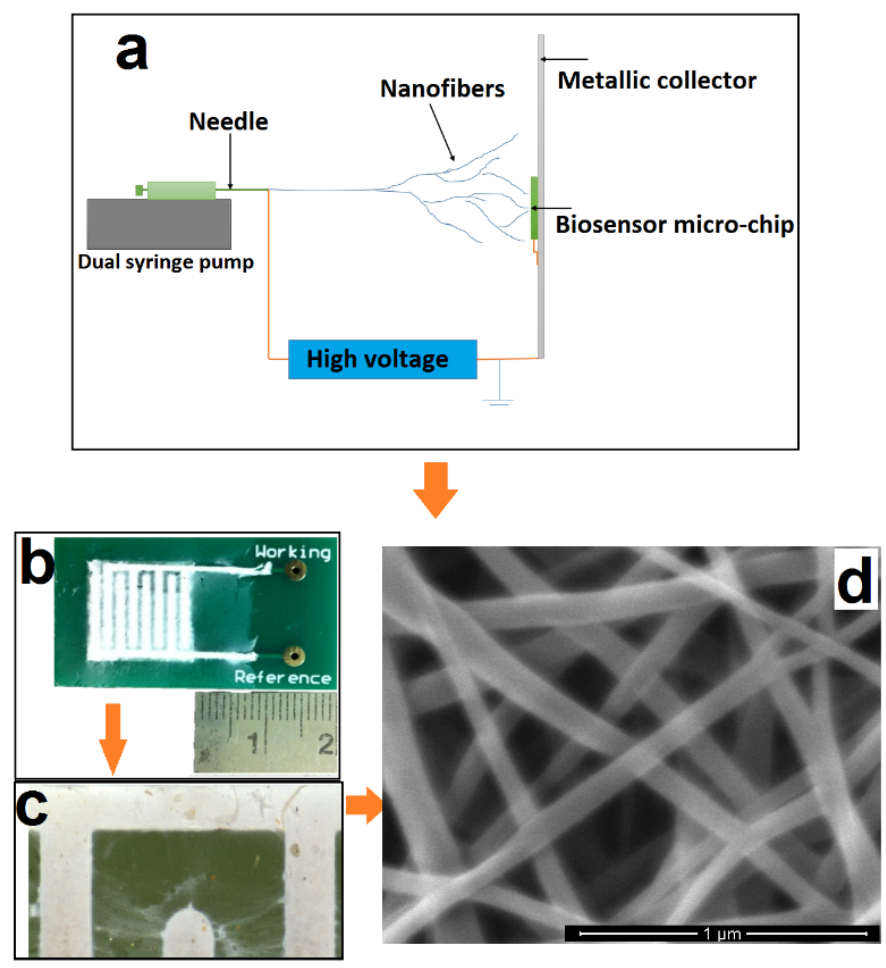

Figure 1. Fabrication of PVA/CNT nanofibers and their deposition on the biosensor: (a) Schematic of nanofiber deposition on the biosensor chip using electrospinning; (b) Optical image of the actual biosensor with selectively deposited nanofibers; (c) Scanning electron microscope (SEM) image of electrospun PVA/CNT nanofibers; (d) High resolution SEM of the deposited nanofibers clearly exhibiting the porous nature of the electrospun nanofibers with a diameter of $\sim 250 \mathrm{~nm}$.

The nanoweb biosensor chip was comprised of a base printed circuit platform. The platform is overlaid with the nanoporous nanoweb layer, which is then encapsulated by microfluidic manifolds manufactured using polydimethylsiloxane for fluid encapsulation and confinement. Based on the SEM images, the area of the pores is estimated to be in the range of 100 to 500 square nanometers. CRP is used in phosphate buffered saline as well as $50 \%$ human serum for evaluating the sensor performance.

Printed circuit board (PCB) chips comprising gold comb shaped designs of dimensions $10 \mathrm{~mm} \times 10 \mathrm{~mm}$ in length; a finger width of $1 \mathrm{~mm}$ with spacings of $1 \mathrm{~mm}$ were manufactured 
with a FR4 passivation layer. After cleaning the surface of these chips with $10 \mathrm{~mL}$ of isopropyl alcohol (IPA) and air drying the samples for $10 \mathrm{~min}$, nanowebs constituting $10 \mathrm{mg}$ of CNT in 10\% PVA were electrospun (Figure 1b). Figure 1c shows a blown-up image of the individual fingers and the presence of the nanoweb in between.

To ensure that the nanofibers are deposited on the gold microelectrodes and not on the FR4 (glass reinforced epoxy laminate printed circuit board) surface of the microchip itself, both the 'Working' and the 'Reference' electrodes were also grounded. Interestingly, the above mentioned procedure results in selective deposition of nanocomposite fibers only on the conductive electrodes allowing the FR4 surface to be clean without any deposition. The average diameter of the nanocomposite fibers is $250 \mathrm{~nm}$, as seen from the SEM image in Figure 1d. Based on the flow rate of $1.5 \mathrm{~mL} / \mathrm{h}$ and the electrospinning time of $\sim 90 \mathrm{~min}$, the thickness of the electrospun nanofibrous substrate was found to be $\sim 0.5 \mathrm{~mm}$. The presence of CNTs in the polymer matrix helps to stretch the nanofibers under the electric field, enabling continuous stretching and thereby forming a uniform fiber diameter without beaded morphology owing to the conductive nature of the CNTs. This ensures uniform fiber distribution, creating a spatially confined environment for better antigen-antibody interactions.

The dimensions of the nanowebs used for the experiments were $13.2 \mathrm{~mm} \times 13.2 \mathrm{~mm}$. The nanowebs were overlaid onto the metallic sensor surface using tweezers. The nanoweb was encapsulated with a polydimethylsiloxane (PDMS) manifold with dimensions of $13 \mathrm{~mm} \times 13 \mathrm{~mm}$. The manifold has a groove of $5 \mathrm{~mm}$ depth to enable the localization of the nanoweb onto the sensor surface. All the steps pertaining to the detection assay were performed on the assembled sensor chip.

Detection of the protein binding event was achieved using electrochemical impedance spectroscopy (EIS), and electrical double layer (EDL) capacitance measurement [9,10,12]. Briefly, the EDL comprises two components: the solution resistance $\left(R_{s}\right)$ and the double layer capacitance $\left(C_{d l}\right)$. The binding of the protein biomolecules to the covalently anchored antibodies in the nanotextured surface resulted in a modulation to the charge at the electrical double layer formed at the solid/liquid interface [12]. This change in charge produced a change in $C_{d l}$. The capacitance change was measured as an impedance change using an impedance analyzer (Impedance/Gain Phase Analyzer, Autolabs, Avon, IN, USA).

Oscillating AC fields of $0.05 \mathrm{~V}$ were applied, and the frequency was scanned over a range of $40 \mathrm{~Hz}-10 \mathrm{kHz}$. Frequencies only of the lower orders (up to $10 \mathrm{kHz}$ ) were considered for this study. At these low frequencies $C_{d l}$ undergoes major variations during protein binding at the electrical interface. The sample volume was maintained throughout the experimental trials at $150 \mu \mathrm{L}$, sufficient to completely wet the sensor surface. This kept the solution resistance $R_{s}$ constant within this frequency regime [10]. This EIS technique produced measurements that were purely the result of the changes in $\mathrm{C}_{\mathrm{dl}}$, and indicated protein binding.

Two sets of dose response experiments were performed to test the capabilities of the nanoweb sensor platform using electrochemical impedance measurements. The first set was focused on detecting CRP when aliquoted in isotonic buffer solution, $0.15 \mathrm{M}$ phosphate buffer saline (PBS). Aliquots of hs-CRP ranging from $1 \mathrm{fg} / \mathrm{mL}$ to $100 \mathrm{ng} / \mathrm{mL}$ were prepared on a logarithmic scale. These concentrations were selected because they represent clinically relevant concentrations of CRP in physiological conditions. It is known that dithiobis succinimidyl propionate (DSP), which is a covalent linker, has a thiol (sulphur) group at one end that has high affinity for the gold surface while the other end, which is the NHS group, binds with the biological macromolecule (anti-CRP in this case) [35,36]. Based on the highly porous structure of the nanofibrous substrate, the DSP linker would permeate through these pores and anchor on the underlying gold substrate. This unique substrate now allows the interaction of the analyte (CRP) with the anti-CRP within these pores, increasing the overall sensitivity of the biosensor. Thus, after integrating the nanoweb mat and PDMS manifold onto the chip, $150 \mu \mathrm{L}$ of $10 \mathrm{mM}$ DSP crosslinker was injected into the manifold and incubated for $30 \mathrm{~min}$ at room temperature. The sensor surface was subsequently washed three times with 0.15 M PBS and baseline PBS measurements were taken. Following the crosslinker deposition, $150 \mu \mathrm{L}$ of $50 \mu \mathrm{g} / \mathrm{mL}$ anti-CRP was incubated onto the sensor surface at $4{ }^{\circ} \mathrm{C}$ for $2 \mathrm{~h}$ to immobilize the receptors. After immobilizing anti-CRP on the sensing surface, 
0.15 M PBS wash was performed three times followed by incubation of super block, a blocking protein that reduces the non-specific binding, on the sensor surface for $30 \mathrm{~min}$ at room temperature to minimize non-specific binding, which was again followed by a 0.15 M PBS wash that was performed three times. A zero dose, corresponding to $0.15 \mathrm{M}$ PBS, was injected into the manifold and the measured impedance was considered as the baseline. All the impedance measurements for different dose concentrations of CRP were normalized to this baseline measurement. Starting from the lowest dose within the range, $150 \mu \mathrm{L}$ of CRP spiked buffer was injected into the manifold, incubated for 15 min and impedance measurement was taken. The change in impedance from baseline measurement was calculated and converted into percentage change of impedance from baseline readings.

The second set of experiments focused on detecting CRP when aliquoted in CRP free human serum (hs). Aliquots of hs-CRP ranging from $1 \mathrm{fg} / \mathrm{mL}$ to $100 \mathrm{ng} / \mathrm{mL}$ were prepared on a logarithmic scale. After immobilization of anti-CRP onto the sensor surface, followed by blocking and washing, the zero dose, corresponding to CRP-free human serum, was injected into the manifold. EIS measurements were taken after $15 \mathrm{~min}$ incubation and considered as the baseline measurement. CRP-spiked human sera of different concentrations were subsequently injected onto the sensor surface and measured impedance was converted to percentage change from baseline impedance.

For finite element analysis, a portion of the microelectrode sensor was taken and electrochemical impedance analysis was carried out to obtain the Nyquist plots. First the area and the volume of the gold surface and the number of gold atoms at the surface of the electrode were calculated by using the concentration of redox couple as $10^{-3} \mathrm{~mol} / \mathrm{m}^{3}$. At the initial stage, only oxidizing agents are present at the electrode surface and, since the electrode is bare, these agents easily get electrons from the gold surface and get reduced. The initial reaction rate, $K_{0}$, at this stage was found to be $\sim 2.5 \times 10^{-4} \mathrm{~cm} / \mathrm{s}$. Utilizing the transport of diluted species module, DSP was diffused on the gold electrode and the surface concentration of DSP was calculated after $1600 \mathrm{~s}$. From the concentration value obtained, it was found that the DSP monolayer occupied $\sim 30 \%$ of the electrode surface with each molecule shielding around 8 gold atoms. According to this insight, $\sim 50 \%$ of the gold atoms at the electrode surface are shielded, which affects the forward reaction rate coefficient, $\mathrm{K}_{\mathrm{f}}$, of the redox reaction and in turn reduces the $\mathrm{K}_{0}$ by another $50 \%$, making it $\sim 1.25 \times 10^{-4} \mathrm{~cm} / \mathrm{s}$. In the same manner, the concentrations of CRP and anti-CRP were also calculated. Considering the size of anti-CRP and CRP, it was found that they could shield around 22 and 62 gold atoms respectively. Thus, $60 \%$ of the gold atoms would be shielded by anti-CRP and $70 \%$ by CRP reducing the $\mathrm{K}_{0}$ by $40 \%\left(1 \times 10^{-4} \mathrm{~cm} / \mathrm{s}\right.$ of the initial value $)$ after anti-CRP deposition and by $30 \%\left(7.5 \times 10^{-5} \mathrm{~cm} / \mathrm{s}\right)$ after CRP deposition. In the case of the nanofiber meshed electrode, we can see from the design that some part of the gold surface is occupied by nanofibers and it was estimated to be covering $\sim 55 \%$ of the gold atoms. Thus, the initial value for $\mathrm{K}_{0}$ was found to be $\sim 1.125 \times 10^{-4} \mathrm{~cm} / \mathrm{s}$. Taking the same assumption for the number of gold atoms shielded by DSP, anti-CRP and CRP, we get the following values of $\mathrm{K}_{0}: 0.5625 \times 10^{-4} \mathrm{~cm} / \mathrm{s}$ after DSP deposition, $0.45 \times 10^{-4} \mathrm{~cm} / \mathrm{s}$ and $0.3375 \times 10^{-4} \mathrm{~cm} / \mathrm{s}$ after anti-CRP and CRP deposition, respectively.

\section{Results}

\subsection{Determination of CRP Using EIS}

The binding of the protein biomolecules to the covalently anchored antibodies on the nanotextured surface results in the modulation of charge at the electrical double layer $\left(C_{d 1}\right)$, as seen in Figure 2. The binding of CRP to its antibody at this interface produces a specific and measurable change of impedance across the electrode. As the binding of the biomolecules occur directly on the sensor surface and is not mediated through a redox probe, the impedance changes are non-inductive in nature.

The charge transfer resistance $\left(R_{c t}\right)$ experienced by the leakage charge through EDL at the sensor/analyte interface and the Warburg impedance $\left(Z_{\mathrm{w}}\right)$, which is the diffusional impedance experienced by ions in bulk buffer, are not dominant at lower frequencies and mostly treated as constant [33]. The solution resistance $\left(R_{S}\right)$ is also considered constant as the analyte volume is kept 
constant throughout the experiments. Therefore, impedance measured at the lower frequencies, i.e., below $1 \mathrm{kHz}$, are representative of the changes to the EDL due to biomolecule binding. Hence, impedance data was represented for $100 \mathrm{~Hz}$, which is a representative frequency of the low frequency range. A higher quantity of target protein in the analyte produces a higher amount of shift in the impedance from baseline and thereby allows for quantitative detection of the protein.

a

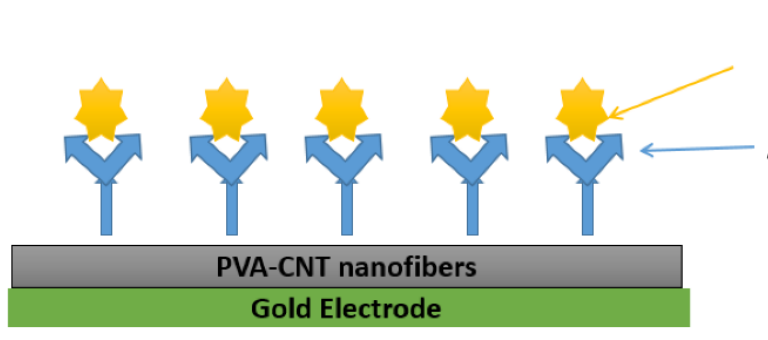

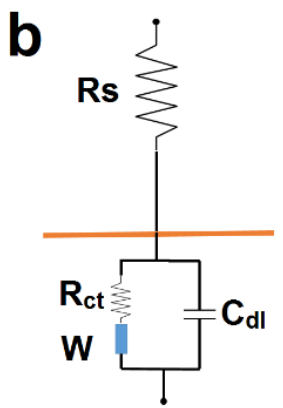

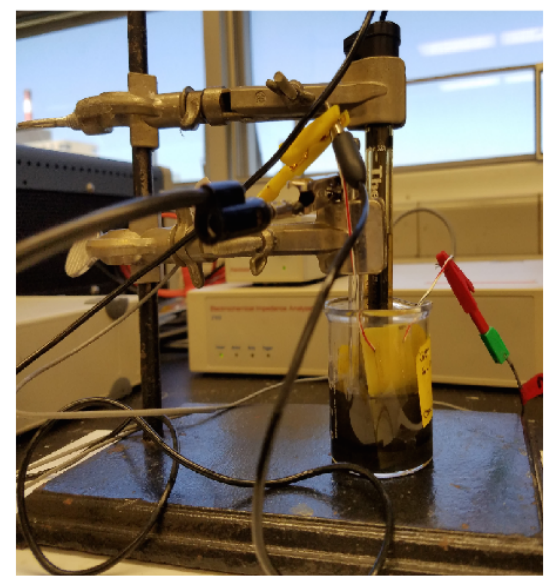

C

Figure 2. General scheme and electrochemical impedance measurement setup: (a) Immobilization of antibody/antigen on the electrospun nanostructured mesh selectively deposited on the interdigitated micro comb gold electrodes; (b) Resulting electrical circuit at the electrode/electrolyte interface; (c) Experimental setup for the quantification of the electrochemical impedance of the PVA/CNT-coated electrode.

A biosensor dose response analysis was performed based on the two sets of experiments with the CRP in PBS buffer and human serum (hs) to test the capabilities of the nanofibrous sensor platform as shown in Figure 3.

As can be seen from Figure 3a, the synthesized nanofibrous sensor platform was able to detect CRP from $100 \mathrm{ng} / \mathrm{mL}$ down to $1 \mathrm{fg} / \mathrm{mL}$, indicating a unique ability of this platform to detect a minimum concentration of CRP. Each concentration was tested on different electrodes to avoid the removal of residual protein and successive regeneration of the sensor surface after every test. Also from Figure $3 b$, comparing the impedance values across this regime between the PBS and hs, it is found that there is relatively larger change in impedance for hs from $\sim 360$ ohms to $\sim 220$ ohms compared to PBS ( 220 ohms to $\sim 140 \mathrm{ohms}$ ), showing that this technique has a lot of potential to detect biological macromolecules in their native environment. 


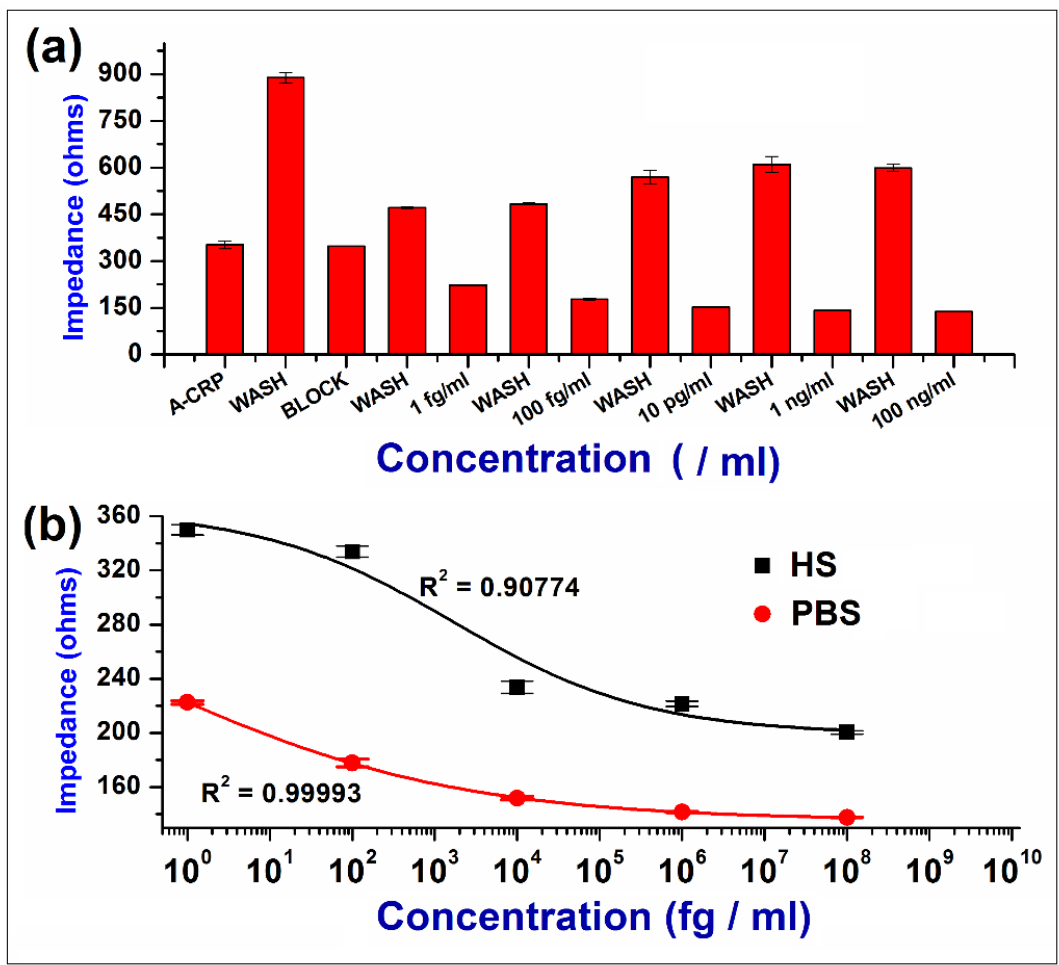

Figure 3. Dose response in PBS and hs: (a) Absolute impedance values after each step of antibody immobilization (A-CRP), followed by washing and superblock (Block) deposition to test the sensitivity of the biosensor on individual electrodes; (b) Changes in the impedance with respect to the concentration of CRP in both PBS and hs. All the impedance measurements are with respect to the concentration of antigen (CRP) per $\mathrm{mL}$ of either buffer (PBS/hs).

\subsection{Biosensor Performance}

The sensor performance was determined by the impedance change from the antibody saturation baseline expressed as a percentage change (Figure 4a,b). As the dose of CRP in the solution increased, the corresponding measured absolute impedance value from the biosensor decreased. Figure 4a shows the percentage change in the impedance with respect to the various doses of CRP in PBS and hs. The limit of detection (LOD) in this case was estimated to be $1 \mathrm{fg} \mathrm{mL} \mathrm{m}^{-1}$ with a percent change of $38 \%$ from the antibody baseline impedance, which is the anti-CRP mounted on the PVA/CNT-coated electrode control. The sensitivity of the sensor was computed by determining the dose of the antigen that gave at least $10 \%$ change from the baseline signal, where $10 \%$ change correlated to the signal background. Figure $4 \mathrm{a}$ also shows the dose response of CRP in 50\% human serum. Initially the background signal from the antibody saturated sensor was determined by injecting $50 \%$ human serum directly on the sensor surface. The assay on the nanofibers was constructed in a similar manner for the detection of CRP from PBS samples. We observed a $45 \%$ change in the case of hs and a $38 \%$ change in the case of PBS for the impedance while detecting the lowest dose of $1 \mathrm{fg} \mathrm{mL}^{-1}$. The percentage change in impedance ranged from $45 \%$ to $70 \%$ (hs) and 38\% to $60 \%$ (PBS) over a concentration range of $1 \mathrm{fg} \mathrm{mL} L^{-1}$ to $100 \mathrm{ng} \mathrm{mL}^{-1}$. Saturation in measurement was observed for the CRP dose of $10 \mathrm{pg} \mathrm{mL}^{-1}$, indicating a limit on linearity from the performance standpoint. 


\section{a}

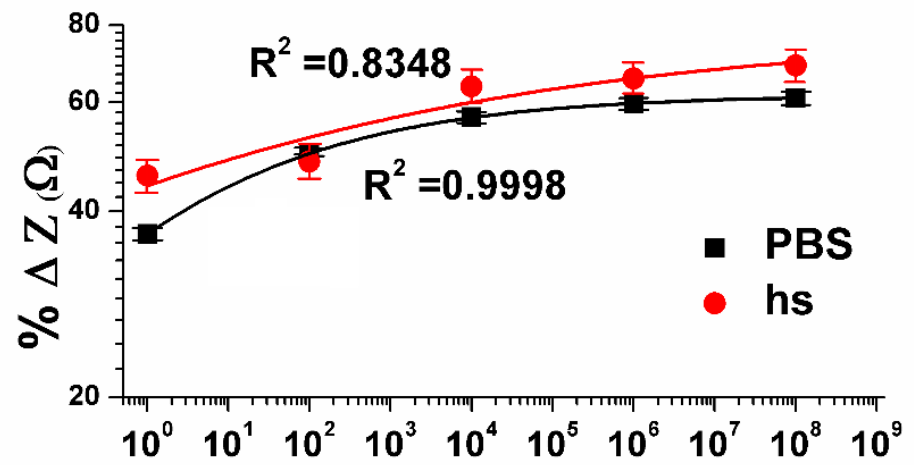

b

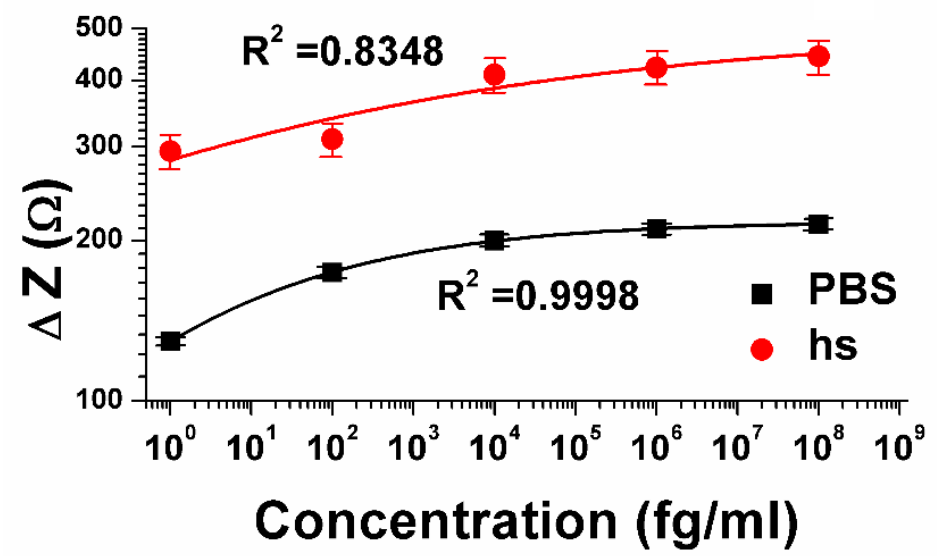

Figure 4. Dose response of CRP in PBS and hs: (a) Percentage change in impedance $(\% \Delta Z)$ corresponding to the concentration of CRP; (b) CRP dose dependent change in the impedance $(\Delta \mathrm{Z})$.

\subsection{Cross-Reactivity Test}

Cross-reactivity tests were performed to determine the selectivity of the biosensor using anti-troponin-T as the antibody and CRP as the antigen. The sensor was immobilized with $1 \mu \mathrm{g} \mathrm{mL}-1$ of anti-troponin- $T$ and was prepared in a manner described earlier for the case of anti-CRP. The cross reactivity was studied by immobilizing anti-troponin-T with CRP in PBS and was compared to the performance of chips with anti-CRP and CRP in both hs and PBS (Figure 5). Dose response studies were conducted in a similar manner to those described in the previous subsection. As before, the impedance response for CRP interaction with anti-CRP ranged from $~ 38 \%$ to $60 \%$ in PBS and $\sim 45 \%$ to $70 \%$ in hs. On the other hand, there was a very low response of the CRP interaction with anti-troponin- $\mathrm{T}$, with the percentage change in impedance below $10 \%$. These results indicated the robustness as well as the selectivity of the designed sensor and its response. 


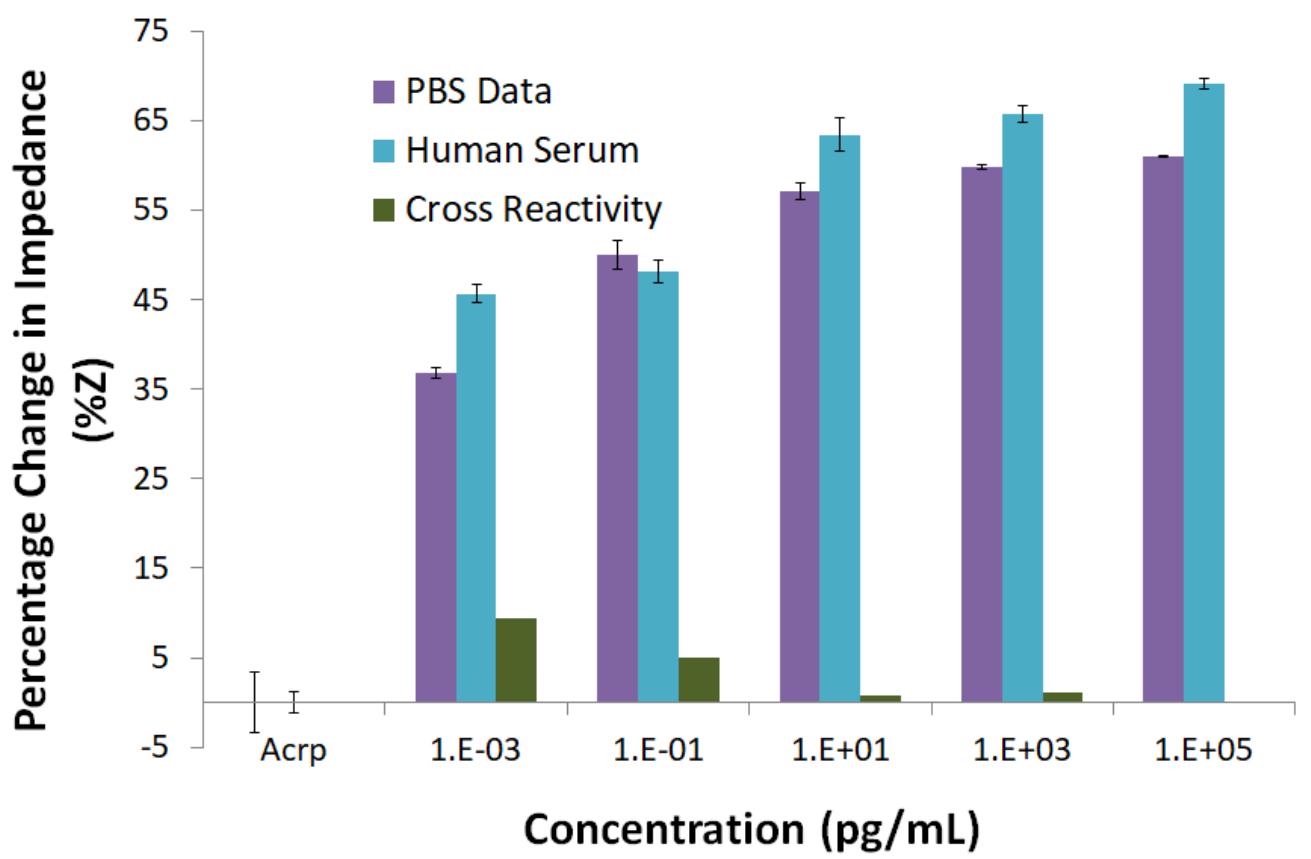

Figure 5. Cross reactivity study. Comparison of CRP binding with anti-CRP in both hs and PBS and cross reactivity of CRP with anti-troponin-T.

\subsection{Finite Element Analysis to Determine the Change in Impedance on Detection of CRP}

Both the electrodes, bare gold and the one covered with nanofiber mesh, were simulated for the change in impedance when depositing DSP, anti-CRP and CRP and were compared with the controls (absence of these molecules). Figure 6a shows the FEA model that was constructed with the same dimensions as the real electrode. Three layers of randomly oriented nanofibers were modeled on the constructed electrode having the same diameter $(\sim 250 \mathrm{~nm})$ as the real nanofibers. As can be seen from Figure $6 b, c$, the Nyquist plots were obtained for both of these scenarios and the change in impedance was compared. It can be seen that a significant change in impedance with deposition of each layer on the bare gold electrode as well as on the nanofiber meshed gold surface was obtained. In addition, it is found that the impedance due to the CRP deposition on bare gold electrodes is less compared to the one with a nanofiber mesh in agreement with the real-time impedance data that was obtained showing the beneficial effects of the nanofiber mesh owing to its porosity. 


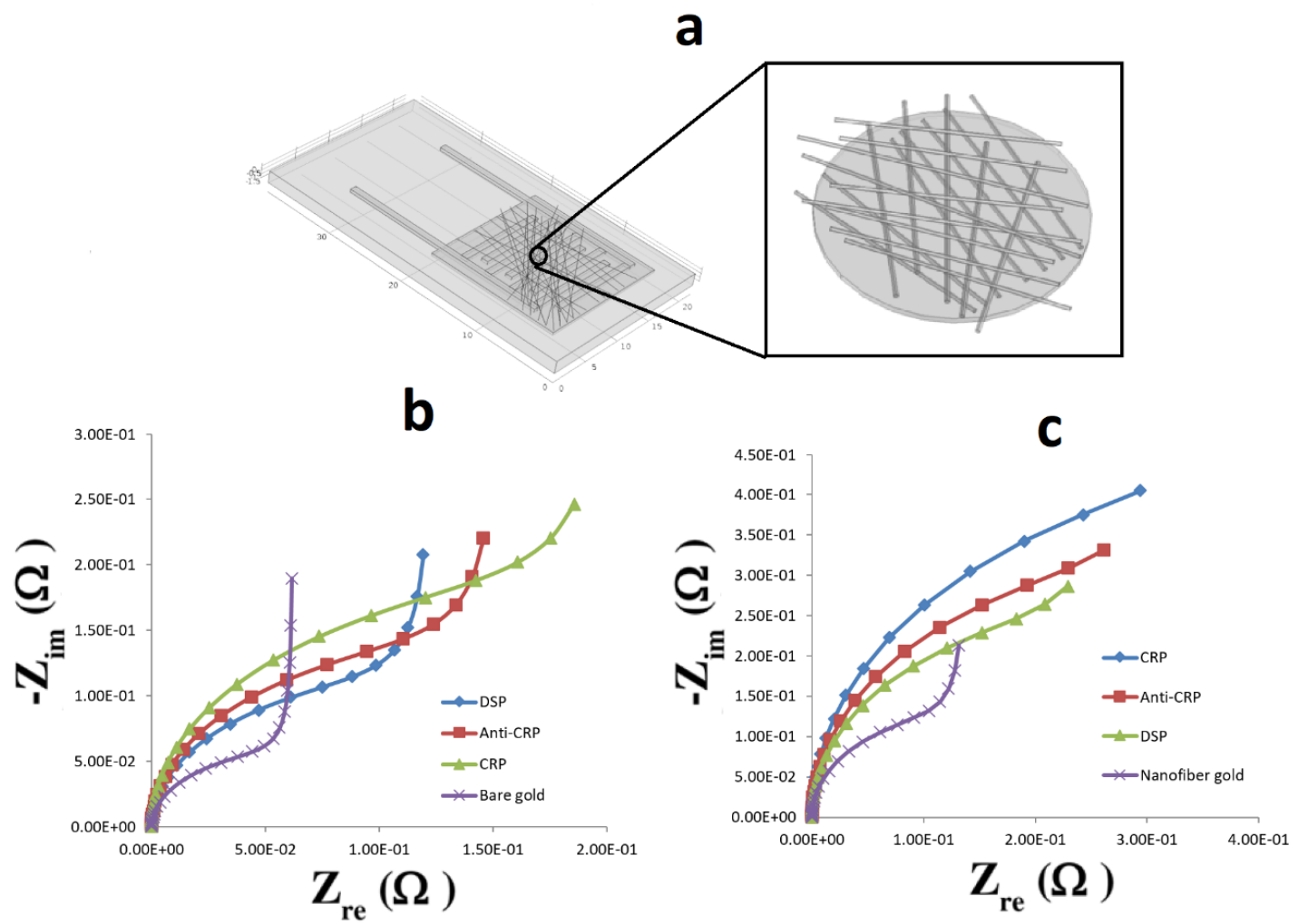

Figure 6. Finite element analysis of the change in impedance due to the presence of CRP, anti-CRP and DSP: (a) Modeled gold coated copper electrode on FR4 material; (b) Change in impedance due to the presence of DSP, anti-CRP and CRP on bare electrodes; (c) Change in impedance due to the presence of DSP, anti-CRP and CRP on nanofiber-coated electrodes.

\section{Discussion}

The PVA/CNT nanofiber-embedded biosensor device exhibits an amplification of the measured electrochemical impedance signal associated with the protein binding. CRP immobilization is achieved using a standard immunoassay protocol. The transduction is chemo-electro ionic with the protein binding resulting in a modulation of the electrical double layer at the interface of the nanofibers. Charged groups are present both on the hydrophilic surface as well as on the hydrophobic residues that are interior to the protein molecule [37]. Upon the application of voltage, the charged surface facilitates either repulsive or attractive forces on the ions at the electrode interface leading to a change in the dielectric thickness across the electrode [34]. These changes in the dielectric thickness are directly proportional to the thickness of the electrical double layer. When the electrode surface becomes nanotextured as in this particular study, the effect of the modulation on the dielectric thickness is amplified primarily due to the segmentation of the electrical double layer.

The changes in the impedance were comparable in both the ionic buffer as well as in human serum. The minimization of high background in the human serum buffer may be attributed to the sieve-like behavior of the nanofibers that provide size matched confinement of the target proteins. Furthermore, given the nature of the substrate, being nanofibrous and porous, it turns out to be a better microenvironment for the analyte to interact. In addition, the pores within the nanofibrous substrate would provide an environment that may promote the phenomenon of macromolecular crowding that typically exists in confined spaces, thereby altering the behavior of human serum [16,17]. Interestingly, it was observed that the protein association is significantly enhanced at lower concentrations, which in turn enhanced the sensitivity of the biosensor device.

The dimensions of the composite nanofibers are $\sim 100 \mu \mathrm{m}$ in length and $\sim 250 \mathrm{~nm}$ in diameter. The nanofibers make a good biochemical transducer due to the presence of CNT and are highly 
suitable for achieving electrical signal amplification associated with the detection of the biomolecules. The resulting nanostructure comprises a non-periodic array of nanoscale confined spaces, which are electrically connected through the metallic micro electrode. The nanofibers have been surface functionalized with a protocol similar to the enzyme linked immunosorbent assay.

The most important characteristic of a biosensor is the calibration dependence of the impedance signal (Z) on the concentration of the analyte (C) [38]. The behavior of the calibration curve can be understood using a suitable mathematical equation such as the Hill isotherm model [39], which is used to describe the binding of different species onto homogenous substrates to fit the experimental data. According to this model, the antigen binding ability at one site on the macromolecule may influence different binding sites on the same macromolecule and the adsorption process is a cooperative phenomenon. As seen in Figure 4, the biosensor response in the form of percent change in impedance $(\% \Delta Z)$ and difference impedance $(\Delta Z)$, are closely fitted with the following Hill formula:

$$
y=\operatorname{Vmax}
$$

where $y$ is the biosensor response (signal Z), Vmax is the maximum state of the reaction reached at concentration $x, k$ is the antigen concentration that binds the receptor sites at half concentration and $n$ is the Hill coefficient.

For Figure 4, the values of $n(0.2221 \pm 0.00374$ (PBS) and $0.1050 \pm 0.0862(\mathrm{hs}))$ are calculated based on Equation (1). This low value of Hill's coefficient at all the concentrations is a measure of the variable free energy of the interactions between biomolecules and surfaces [40-42]. In both PBS and hs, Hill's slope is less than 1, which is an indication of negative cooperative binding, which means that although many binding sites may be present, only specific binding occurs at a given point of time [43]. This observation is also supported by the cross-reactivity study, where at $1 \mathrm{fg} \mathrm{mL}-1$ concentration of CRP, the percentage change in the impedance is less than $10 \%$. The values of $k$ are $0.1744 \pm 0.0089 \mathrm{fg} \mathrm{mL}^{-1}$ (PBS) and $0.0708 \pm 0.2357 \mathrm{fg} \mathrm{mL}^{-1}$ (hs), respectively.

\section{Conclusions}

A novel biosensor device is fabricated and tested for the detection of CRP protein. This affinity-based biosensor, working on the principle of electrochemical impedance spectroscopy, demonstrated an excellent signal to noise ratio with a detection limit of $1 \mathrm{fg} \mathrm{mL}^{-1}$. Furthermore, a $45 \%$ (hs) and 38\% (PBS) change in the electrical impedance was observed while measuring the lowest detectable dose of $1 \mathrm{fg} \mathrm{mL}^{-1}$. It is proposed that within the confinement of the pores of the nanofibrous mesh, the overall specific binding increases, thereby reducing the noise set by non-specific interactions. The signal transduction is chemo-electro ionic with the protein binding resulting in a modulation of the electrical double layer capacitance at the nanofibrous interface. Furthermore, the simulated behavior of the biosensor electrode in the presence and absence of the nanofiber mesh clearly demonstrated a larger change in impedance owing to the nanofiber mesh indicating the role of porosity in the detection of CRP.

Author Contributions: Conceptualization, I.M., A.A. and P.P.; Methodology, A.A., S.P. and P.P.; Software, I.M. and P.B.; Validation, I.M., A.A. and P.B.; Formal analysis, I.M., P.B. and A.A.; Investigation, I.M. and A.A.; Resources, S.P. and P.P.; Data curation, I.M. and A.A.; Writing—original draft preparation, I.M. and A.A.; Writing—review and editing, I.M.; Supervision, S.P. and P.P.; Project administration, I.M., A.A. and P.P. All authors have read and agreed to the published version of the manuscript.

Funding: This research received no external funding.

Conflicts of Interest: The authors declare no conflict of interest. 


\section{References}

1. Schiess, R.; Wollscheid, B.; Aebersold, R. Targeted proteomic strategy for clinical biomarker discovery. Mol. Oncol. 2009, 3, 33-44. [CrossRef] [PubMed]

2. Hage, F.G.; McCrory, M.; Szalai, A. C-reactive protein and cardiovascular disease: Lessons learned from studying genetically engineered mice. In C-Reactive Protein: New Research; Nagasawa, S., Ed.; Nova Publishers: Hauppauge, NY, USA, 2009; pp. 83-116. ISBN 978-1-60692-237-8.

3. Hage, F.G. C-reactive protein and hypertension. J. Hum. Hypertens. 2014, 28, 410-415. [CrossRef] [PubMed]

4. Nagai, T.; Anzai, T.; Kaneko, H.; Mano, Y.; Anzai, A.; Maekawa, Y.; Takahashi, T.; Meguro, T.; Yoshikawa, T.; Fukuda, K. C-reactive protein overexpression exacerbates pressure overload-induced cardiac remodeling through enhanced inflammatory response. Hypertension 2011, 57, 208-215. [CrossRef] [PubMed]

5. Benjamin, E.J.; Blaha, M.J.; Chiuve, S.E.; Cushman, M.; Das, S.R.; Deo, R.; de Ferranti, S.D.; Floyd, J.; Fornage, M.; Gillespie, C.; et al. Heart disease and stroke statistics-2017 update: A report from the american heart association. Circulation 2017, 135, e146-e603.

6. Peng, G.; Et, A. Detection of lung, breast, colorectal, and prostate cancers from exhaled breath using a single array of nanosensors. Br. J. Cancer 2010, 103, 524-551. [CrossRef]

7. Koh, I.; Josephson, L. Magnetic nanoparticle sensors. Sensors 2009, 9, 8130-8145. [CrossRef]

8. Engel, Y.; Elnathan, R.; Pevzner, A.; Davidi, G.; Flaxer, E.; Patolsky, F. Supersensitive detection of explosives by silicon nanowire arrays. Angew. Chemie Int. Ed. 2010, 49, 6830-6835. [CrossRef]

9. Dan, Y.; Lu, Y.; Kybert, N.J.; Luo, Z.; Johnson, A.T.C. Intrinsic response of graphene vapor sensors. Nano Lett. 2009, 9, 1472-1475. [CrossRef]

10. Vance, S.A.; Sandros, M.G. Zeptomole detection of C-reactive protein in serum by a nanoparticle amplified surface plasmon resonance imaging aptasensor. Sci. Rep. 2014, 4, 1-7. [CrossRef]

11. Fakanya, W.; Tothill, I. Detection of the inflammation biomarker c-reactive protein in serum samples: Towards an optimal biosensor formula. Biosensors 2014, 4, 340-357. [CrossRef] [PubMed]

12. Ridker, P. C-reactive protein a simple test to help predict risk of heart attack and stroke. Circulation 2003, 108, 81-85. [CrossRef] [PubMed]

13. Ridker, P.M. Clinical application of C-reactive protein for cardiovascular disease detection and prevention. Circulation 2003, 107, 363-369. [CrossRef] [PubMed]

14. El-Moghazy, A.Y.; Huo, J.; Amaly, N.; Vasylieva, N.; Hammock, B.D.; Sun, G. An innovative nanobody-based electrochemical immunosensor using decorated nylon nanofibers for point-of-care monitoring of human exposure to pyrethroid insecticides. ACS Appl. Mater. Interfaces 2020, 12, 6159-6168. [CrossRef] [PubMed]

15. El-Moghazy, A.Y.; Zhao, C.; Istamboulie, G.; Amaly, N.; Si, Y.; Noguer, T.; Sun, G. Ultrasensitive label-free electrochemical immunosensor based on PVA-co-PE nanofibrous membrane for the detection of chloramphenicol residues in milk. Biosens. Bioelectron. 2018, 117, 838-844. [CrossRef]

16. Zimmerman, S.B.; Minton, A.P. Macromolecular Crowding: Biochemical, biophysical, and physiological consequences. Annu. Rev. Biophys. Biomol. Struct. 1993, 22, 27-65. [CrossRef]

17. Ellis, R.J. Macromolecular crowding: Obvious but underappreciated. Trends Biochem. Sci. 2001, 26, 597-604. [CrossRef]

18. Dobson, C.M. Chemical space and biology. Nature 2004, 432, 824-828. [CrossRef]

19. Zimmerman, S.B.; Trach, S.O. Estimation of macromolecule concentrations and excluded volume effects for the cytoplasm of Escherichia coli. J. Mol. Biol. 1991, 222, 599-620. [CrossRef]

20. Kosaka, P.M.; Tamayo, J.; Ruz, J.J.; Puertas, S.; Polo, E.; Grazu, V.; de la Fuente, J.M.; Calleja, M. Tackling reproducibility in microcantilever biosensors: A statistical approach for sensitive and specific end-point detection of immunoreactions. Analyst 2013, 138, 863-872. [CrossRef]

21. Nair, P.R.; Alam, M.A. Performance limits of nanobiosensors. Appl. Phys. Lett. 2006, 88, 233120. [CrossRef]

22. Kosaka, P.M.; Pini, V.; Ruz, J.J.; da Silva, R.A.; González, M.U.; Ramos, D.; Calleja, M.; Tamayo, J. Detection of cancer biomarkers in serum using a hybrid mechanical and optoplasmonic nanosensor. Nat. Nanotechnol. 2014, 9, 1047-1053. [CrossRef] [PubMed]

23. Froehling, P.E. Dendrimers and dyes-A review. Dye. Pigment. 2001, 48, 187-195. [CrossRef]

24. Evanko, D. Label-free microscopy. Nat. Methods 2010, 7, 36. [CrossRef]

25. Sadik, O.A.; Aluoch, A.O.; Zhou, A. Status of biomolecular recognition using electrochemical techniques. Biosens. Bioelectron. 2009, 24, 2749-2765. [CrossRef] [PubMed] 
26. Ma, H.; Wallbank, R.W.R.; Chaji, R.; Li, J.; Suzuki, Y.; Jiggins, C.; Nathan, A. An impedance-based integrated biosensor for suspended DNA characterization. Sci. Rep. 2013, 3, 2730. [CrossRef]

27. Maruyama, Y.; Terao, S.; Sawada, K. Label free CMOS DNA image sensor based on the charge transfer technique. Biosens. Bioelectron. 2009, 24, 3108-3112. [CrossRef]

28. Rothberg, J.M.; Hinz, W.; Rearick, T.M.; Schultz, J.; Mileski, W.; Davey, M.; Leamon, J.H.; Johnson, K.; Milgrew, M.J.; Edwards, M.; et al. An integrated semiconductor device enabling non-optical genome sequencing. Nature 2011, 475, 348-352. [CrossRef]

29. Gonçalves, D.; Prazeres, D.M.F.; Chu, V.; Conde, J.P. Label-free electronic detection of biomolecules using a-Si:H field-effect devices. J. Non. Cryst. Solids 2006, 352, 2007-2010. [CrossRef]

30. Kim, N.-Y.; Adhikari, K.K.; Dhakal, R.; Chuluunbaatar, Z.; Wang, C.; Kim, E.-S. Rapid, sensitive, and reusable detection of glucose by a robust radiofrequency integrated passive device biosensor chip. Sci. Rep. 2015, 5, 7807. [CrossRef]

31. Barbaro, M.; Bonfiglio, A.; Raffo, L.; Alessandrini, A.; Facci, P.; BarakBarak, I. A CMOS, fully integrated sensor for electronic detection of DNA hybridization. IEEE Electron Device Lett. 2006, 27, 595-597. [CrossRef]

32. Stagni, C.; Guiducci, C.; Benini, L.; Ricco, B.; Carrara, S.; Paulus, C.; Schienle, M.; Thewes, R. A Fully Electronic Label-Free DNA Sensor Chip. IEEE Sens. J. 2007, 7, 577-585. [CrossRef]

33. Sun, T.; Swindle, E.J.; Collins, J.E.; Holloway, J.A.; Davies, D.E.; Morgan, H. On-chip epithelial barrier function assays using electrical impedance spectroscopy. Lab Chip 2010, 10, 1611-1617. [CrossRef] [PubMed]

34. Daniels, J.S.; Pourmand, N. Label-free impedance biosensors: Opportunities and challenges. Electroanalysis 2007, 19, 1239-1257. [CrossRef] [PubMed]

35. Balamurugan, S.; Obubuafo, A.; Soper, S.A.; Spivak, D.A. Surface immobilization methods for aptamer diagnostic applications. Anal. Bioanal. Chem. 2008, 390, 1009-1021. [CrossRef] [PubMed]

36. Riepl, M.; Mirsky, V.M.; Novotny, I.; Tvarozek, V.; Rehacek, V.; Wolfbeis, O.S. Optimization of capacitive affinity sensors: Drift suppression and signal amplification. In Proceedings of the Analytica Chimica Acta; Elsevier: Amsterdam, The Netherlands, 1999; Volume 392, pp. 77-84.

37. Gitlin, I.; Carbeck, J.D.; Whitesides, G.M. Why are proteins charged? Networks of charge-charge interactions in proteins measured by charge ladders and capillary electrophoresis. Angew. Chem. Int. Ed. Engl. 2006, 45, 3022-3060. [CrossRef]

38. Kurganov, B.; Lobanov, A.; Borisov, I.; Reshetilov, A. Criterion for hill equation validity for description of biosensor calibration curves. Anal. Chim. Acta 2001, 427, 11-19. [CrossRef]

39. Hill, A. The possible effects of the aggregation of the molecules of haemoglobin on its dissociation curves. J. Physiol. 1910, 40, 4-7.

40. Endrenyi, L.; Fajszi, C.; Kwong, F.H. Evaluation of Hill slopes and Hill coefficients when the saturation binding or velocity is not known. Eur. J. Biochem. 1975, 51, 317-328. [CrossRef]

41. Wyman, J. Linked functions and reciprocal effects in hemoglobin: A second look. Adv. Protein Chem. 1964, 19, 223-286.

42. Saroff, H.A.; Minton, A.P. The Hill plot and the energy of interaction in hemoglobin. Science 1972, 175, 1253-1255. [CrossRef]

43. Kirsch, J.F. Enzyme kinetics and mechanism, by Paul F. Cook and WW Cleland. Protein Sci. 2008, 17, 380-381. [CrossRef]

(C) 2020 by the authors. Licensee MDPI, Basel, Switzerland. This article is an open access article distributed under the terms and conditions of the Creative Commons Attribution (CC BY) license (http://creativecommons.org/licenses/by/4.0/). 